\title{
Pion Electromagnetic Form Factor in Virtuality Distribution Formalism
}

\section{A. V. Radyushkin *}

Physics Department, Old Dominion University, Norfolk, VA 23529, USA

Thomas Jefferson National Accelerator Facility, Newport News, VA 23606, USA

E-mail: radyush@jlab.org

We discuss two applications of the Virtuality Distribution Amplitudes (VDA) formalism developed in our recent papers. We start with an overview of the main properties of the pion distribution amplitude emphasizing the quantitative measures of its width, and possibility to access them through the pion transition form factor studies. We formulate the basic concepts of the VDA approach and introduce the pion transverse momentum distribution amplitude (TMDA) which plays, in a covariant Lagrangian formulation, a role similar to that of the pion wave function in the 3-dimensional Hamiltonian light-front approach. We propose simple factorized models for soft TMDAs, and use them to describe existing data on the pion transition form factor, thus fixing the scale determining the size of the transverse-momentum effects. Finally, we apply the VDA approach to the one-gluon exchange contribution for the pion electromagnetic form factor. We observe a very late $Q^{2} \gtrsim 20 \mathrm{GeV}^{2}$ onset of transition to the asymptotic pQCD predictions and show that in the $Q^{2} \lesssim 10 \mathrm{GeV}^{2}$ region there is essentially no sensitivity to the shape of the pion distribution amplitude. Furthermore, the magnitude of the one-gluon exchange contribution in this region is estimated to be an order of magnitude below the Jefferson Lab data, thus leaving the Feynman mechanism as the only one relevant to the pion electromagnetic form factor behavior for accessible $Q^{2}$.

QCD Evolution 2015 -QCDEV2015-

26-30 May 2015

Jefferson Lab (JLAB), Newport News Virginia, USA

\footnotetext{
* Speaker.
} 


\section{Introduction}

Adding transverse momentum degrees of freedom is a popular subject in the theory of hard reactions. In reality, such an adding is not always necessary. We mean that when the standard ("collinear") perturbative QCD (pQCD) factorization works, one deals with objects like usual parton densities (for inclusive processes) and hadron distribution amplitudes (for exclusive processes) none of which explicitly depends on transverse momenta. Explanations "to public" that these functions result from integrating some more general transverse momentum-dependent functions over $k_{\perp}$ may help to create an intuitive picture, but in fact all the functions entering into the collinear factorization formulas are defined without any reference to transverse momentum, say, through matrix elements of bilocal operators on the light cone. Still, it might be helpful to have "underlying" formulas, in which transverse momentum enters explicitly.

An important example is the pion distribution amplitude, which is usually [1] defined through a matrix element of a bilocal operator on the light cone, but sometimes [2] is introduced through the light-front wave function $\psi\left(x, k_{\perp}\right)$ integrated over transverse momentum $k_{\perp}$. A subtle point is that the first definition is made within the operator product expansion (OPE) formalism of the covariant 4-dimensional Lagrangian quantum field theory (QFT), while the wave function $\psi\left(x, k_{\perp}\right)$ is an object of a very different Hamiltonian 3-dimensional light-front (LF) approach [3, 4].

In our recent papers $[5,6,7,8]$, we have developed a formalism which is based on a covariant QFT in 4 dimensions, but still manages to encode information about the hadronic structure in hard exclusive processes in terms of transverse momentum dependent distribution amplitudes (TMDAs) $\Psi\left(x, k_{\perp}\right)$ that incorporate the dependence on the transverse momentum of its constituents. Just like in the light-front formalism, the organization of these functions has the structure of the Fock state decomposition, i.e. each function is characterized by the number of constituents involved.

For exclusive processes, a standard situation asking to add transverse momentum degrees of freedom is when the collinear factorization integral diverges at the end point, and hence one needs some natural source of a cut-off. In this talk, we discuss the use of the TMDA formalism in the description of the photon-pion transition and pion electromagnetic form factors.

\section{Pion Distribution Amplitude}

Within the covariant QFT, the pion distribution amplitude (DA) $\varphi_{\pi}(x)$ is introduced [1] as a function whose $x^{N}$ moments $f_{N}(\mu)$ are given by matrix elements of twist-2 local operators

$$
f_{N}(\mu)=\int_{0}^{1} x^{N} \varphi_{\pi}(x, \mu) d x, i^{N+1}\left\langle 0\left|\bar{d}(0) \gamma_{5} \not h(n D)^{N} u(0)\right| \pi^{+}, P\right\rangle=(P n)^{N+1} f_{N}(\mu)
$$

with $n^{2}=0$ and $\mu$ being the UV renormalization scale for operators. Since the zeroth moment,

$$
\int_{0}^{1} \varphi_{\pi}(x, \mu) d x=f_{\pi}
$$

is given by the pion decay constant $f_{\pi}$, we have an important constraint on the pion DA, fixing the integral under the $\varphi_{\pi}(x)$ curve, but it puts no restrictions on its shape. In fact, the pion DA depends 
on the renormalization scale $\mu: \varphi_{\pi}(x) \rightarrow \varphi_{\pi}(x, \mu)$. The solution of the evolution equation for the pion DA was obtained $[9,2]$ in the form of expansion over Gegenbauer polynomials

$$
\varphi_{\pi}(x, \mu)=6 f_{\pi} x(1-x)\left\{1+\sum_{n=1}^{\infty} a_{2 n} C_{2 n}^{3 / 2}(2 x-1)\left[\ln \left(\mu^{2} / \Lambda^{2}\right)\right]^{-\gamma_{2 n} / \beta_{0}}\right\} .
$$

Since $\gamma_{2 n}>0$, the pion DA acquires a simple form [10] $\varphi_{\pi}(x, \mu \rightarrow \infty)=6 f_{\pi} x(1-x)$ (known as the "asymptotic DA") when the normalization scale $\mu$ tends to infinity.

A quantitative measure of the width of the pion DA at low normalization scales $\mu \lesssim 1 \mathrm{GeV}$ is given by its moments $\left\langle\xi^{2}\right\rangle$ and $\left\langle\xi^{4}\right\rangle$ in the relative variable $\xi \equiv x-(1-x)$. Namely,

$\left\langle\xi^{2}\right\rangle=0$ for infinitely narrow DA $\varphi_{\pi}^{\text {narrow }}(x)=f_{\pi} \delta(x-1 / 2)$;

$\left\langle\xi^{2}\right\rangle=1 / 5$ for asymptotic DA $\varphi_{\pi}(x)=6 f_{\pi} x(1-x)$;

$\left\langle\xi^{2}\right\rangle=1 / 4$ for "root" DA $\varphi_{\pi}(x)=\frac{8}{\pi} f_{\pi} \sqrt{x(1-x)}$; and $\left\langle\xi^{2}\right\rangle=1 / 3$ for flat DA $\varphi_{\pi}(x)=f_{\pi}$.


Figure 1: Shapes for pion distribution amplitude. On the left: narrow (green), asymptotic (red), flat (blue). On the right: asymptotic (red), flat (green), Chernyak-Zhitnitsky (blue).

In particular, a QCD sum rule calculation of Chernyak and Zhitnitsky (CZ) gave the result $\left\langle\xi^{2}\right\rangle=0.40$ (at $\mu^{2}=1.5 \mathrm{GeV}^{2}$ ), which is larger than the value $1 / 3$ of flat DA. The fitting model $\varphi_{\pi}^{\mathrm{CZ}}(x)=30 f_{\pi} x(1-x)(1-2 x)^{2}$ was constructed from the sum of two first terms of the Gegenbauer expansion (2.3), which has $x(1-x)$ as an overall factor, thus excluding all models with DA's that do not linearly vanish at the end-points. However, there is no a priori principle justifying such a requirement: it is just an assumption which may or may not be true.

One can rewrite QCD sum rule for DA itself rather than for its moments:

$$
f_{\pi} \varphi_{\pi}(x)=\frac{3 M^{2}}{2 \pi^{2}}\left(1-e^{-s_{0} / M^{2}}\right) x(1-x)+\frac{8}{81} \frac{\pi \alpha_{s}\langle\bar{q} q\rangle^{2}}{M^{4}}\left\{11 \delta(x)+2 \delta^{\prime}(x)+\{x \rightarrow 1-x\}\right\},
$$

demonstrating that the widening of the pion DA in $\mathrm{CZ}$ calculation is produced by delta-function terms due to the quark and gluon condensates taken in local approximation. Bringing in the nonlocality of condensates changes $\delta(x) \rightarrow 2 x \theta(x<\Delta) / \Delta^{2}$ with $\Delta=\lambda_{q}^{2} / 2 M^{2}$ and $\lambda_{q}^{2}$ fixed from the ratio $\lambda_{q}^{2}=\left\langle\bar{q} D^{2} q\right\rangle /\langle\bar{q} q\rangle \approx 0.4 \mathrm{GeV}^{2}$. This modification [11, 12] decreases resulting $\left\langle\xi^{2}\right\rangle$ to $\approx 0.25$. This value (obtained almost 30 years ago) is in a complete agreement with the most recent lattice result [13]: $\left\langle\xi^{2}\right\rangle=0.24$ at $\mu=2 \mathrm{GeV}$. The "root" model

$$
\varphi_{\pi}^{\text {root }}(x)=\frac{8}{\pi} f_{\pi} \sqrt{x(1-x)}
$$

producing $\left\langle\xi^{2}\right\rangle=\frac{1}{4}$ was proposed in our 1986 paper [11]. The magnitude of this DA in the middle $\varphi_{\pi}^{\text {root }}(1 / 2) / f_{\pi}=4 / \pi \approx 1.27$ is close to the $\approx 1.2$ value found by Braun and Filyanov in 1988 [14]. 


\section{Photon-pion transition form factor}

There is also a hope that information about the shape of pion DA can be extracted from experimental data, in particular from the form factor $F_{\gamma^{*} \gamma^{*} \pi^{0}}\left(q_{1}^{2}, q_{2}^{2}\right)$. If one of the photons is real, the

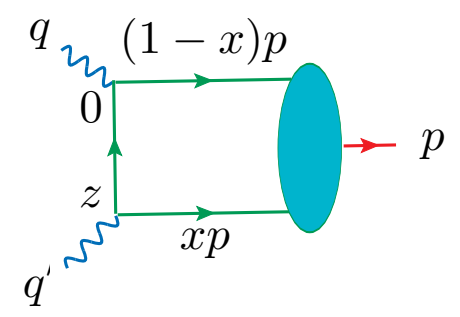

Figure 2: Parton picture for handbag diagram describing photon-pion transition form factor.

leading-order perturbative QCD (pQCD) prediction [4] is

$$
F_{\gamma^{*} \gamma \pi}^{\mathrm{pQCD}}\left(Q^{2}\right)=\frac{\sqrt{2}}{3 Q^{2}} \int_{0}^{1} \frac{\varphi_{\pi}(x)}{x} d x \equiv \frac{\sqrt{2} f_{\pi}}{3 Q^{2}} J .
$$

Information about the shape of the pion DA is now accumulated in the factor $J$. Namely, $J^{\text {narrow }}=2$ for the infinitely narrow DA, $\varphi^{\text {narrow }}(x)=f_{\pi} \delta(x-1 / 2)$;

$J^{\text {as }}=3$ for asymptotic DA $\varphi^{\text {as }}(x)=6 f_{\pi} x(1-x)$,

$J^{\text {root }}=4$ for "root" DA $\varphi_{\pi}(x)=\frac{8}{\pi} f_{\pi} \sqrt{x(1-x)}$, while

$J^{\mathrm{CZ}}=5$ for the $\mathrm{CZ}$ model $\varphi^{\mathrm{CZ}}(x)=30 f_{\pi} x(1-x)(1-2 x)^{2}$.

Experimentally, $F_{\gamma^{*} \gamma^{*} \pi^{0}}\left(q_{1}^{2} \approx 0, q_{2}^{2}=-Q^{2}\right)$ was measured recently by BABAR [15] and Belle [16] collaborations. Unexpectedly, BABAR data [15] are well described by a logarithmic function [17]

$$
J^{L}\left(Q^{2}\right)=\ln \left(\frac{Q^{2}}{M^{2}}+1\right)=Q^{2} \int_{0}^{1} \frac{d x}{x Q^{2}+M^{2}},
$$

if one takes $M^{2}=0.6 \mathrm{GeV}^{2}$. Notice that $J^{L}\left(Q^{2}\right)$ can be obtained if one uses a flat DA $\varphi_{\pi}(x)=f_{\pi}$ and changes $x Q^{2} \rightarrow x Q^{2}+M^{2}$ in the pQCD expression (3.1), expecting that $M^{2}$ is generated by transverse momentum. However, the $1 / x Q^{2} \rightarrow 1 /\left(x Q^{2}+M^{2}\right)$ modification is bringing in, before the integration over $x$, a tower of higher twist $\left(M^{2} / x Q^{2}\right)^{n}$ power corrections. But it is known [18]
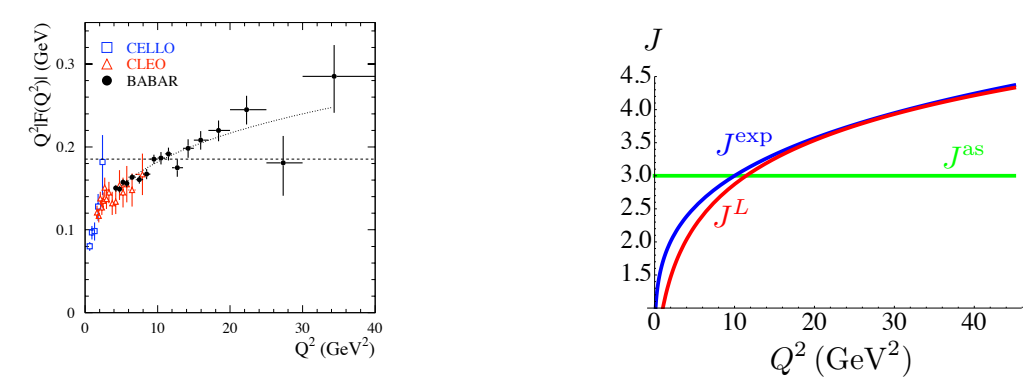

Figure 3: Left: BABAR data. Right: Fit $J^{\exp }\left(Q^{2}\right)$ shown with asymptotic prediction $J^{\text {as }}=3$ and $J^{L}\left(Q^{2}\right)$. 
that the handbag diagram, because of its simple singularity structure near the coordinate light cone $z^{2}=0$, cannot generate an infinite tower of power corrections: there are just two power terms in the OPE for the handbag contribution, those generated by twist- 2 and twist- 4 operators.

\section{TMDA expression for handbag amplitude}

To correctly include transverse momentum effects, we parametrize the relevant matrix element (taking scalar case to simplify notations, for spin-1/2 quarks formulas are the same unless stated)

$$
\langle p|\phi(0) \phi(z)| 0\rangle \equiv F\left((p z), z^{2}\right)=\int_{0}^{\infty} d \sigma \int_{0}^{1} d x \Phi(x, \sigma) e^{i x(p z)-i \sigma\left(z^{2}-i \varepsilon\right) / 4}
$$

through the virtuality distribution amplitude (VDA) $\Phi(x, \sigma)$. After that, the coordinate $d^{4} z$ integral (see Fig. 2) can be taken, and we get the handbag diagram contribution in the VDA representation

$$
T\left(Q^{2}\right)=\int_{0}^{1} \frac{d x}{x Q^{2}} \int_{0}^{\infty} d \sigma \Phi(x, \sigma)\left\{1-e^{-\left[i x Q^{2}+\varepsilon\right] / \sigma}\right\} .
$$

The first term here corresponds to the twist-2 approximation, with the $\sigma$-integral of $\Phi(x, \sigma)$ giving the pion DA $\varphi_{\pi}(x)$. The second term combines the contributions of "invisible" higher-twist operators uncapable to produce power corrections. However, after integration over $\sigma$ this term produces a nontrivial function of $x Q^{2}$. To interpret this result in terms of transverse momentum, we first specify that the pion momentum $p$ has no transverse components becoming pure $p^{+}$for $p^{2}=0$, and then project the matrix element on $z^{+}=0$ plane by taking $z=\left(z^{-}, z_{\perp}\right)$

$$
\left.\langle p|\phi(0) \phi(z)| 0\rangle\right|_{z^{+}=0, p_{\perp}=0}=\int_{0}^{1} d x \varphi\left(x, z_{\perp}\right) e^{i x\left(p z^{-}\right)} .
$$

This defines the impact parameter distribution amplitude (IDA) $\varphi\left(x, z_{\perp}\right)$ whose $z_{\perp}$ Fourier transform gives the transverse momentum dependent distribution amplitude (TMDA) $\Psi\left(x, k_{\perp}\right)$

$$
\varphi\left(x, z_{\perp}\right)=\int \Psi\left(x, k_{\perp}\right) e^{i\left(k_{\perp} z_{\perp}\right)} d^{2} k_{\perp}=\int_{0}^{\infty} d \sigma \Phi(x, \sigma) e^{i \sigma\left(z_{\perp}^{2}+i \varepsilon\right) / 4} .
$$

The crucial fact is that TMDA can be written in terms of the covariantly defined VDA

$$
\Psi\left(x, k_{\perp}\right)=\frac{i}{\pi} \int_{0}^{\infty} \frac{d \sigma}{\sigma} \Phi(x, \sigma) e^{-i\left(k_{\perp}^{2}-i \varepsilon\right) / \sigma} .
$$

As a result, the handbag term may be written in terms of TMDA. For spin-1/2 quarks, it reads

$$
T\left(Q^{2}\right)=\int_{0}^{1} \frac{d x}{x Q^{2}} \int_{k_{\perp}^{2} \leq x Q^{2}} \Psi\left(x, k_{\perp}\right)\left[1-\frac{k_{\perp}^{2}}{x Q^{2}}\right] d^{2} k_{\perp} .
$$

One can see here the twist-2 and twist-4 terms as explicit power-like contributions. The hidden higher twist contributions correspond then to the (minus) integral over the region $k_{\perp}^{2} \geq x Q^{2}$. Now, if $\Psi\left(x, k_{\perp}\right)$ decreases faster than any power of $1 / k_{\perp}^{2}$ for large $k_{\perp}$ (a usual assumption for the nonperturbative part of $\left.\Psi\left(x, k_{\perp}\right)\right)$, then the corrections to the twist-2 and twist-4 term inside the integral over $x$ would decrease faster than any power of $1 / Q^{2}$ for large $Q^{2}$, i.e. they have the property expected from "invisible" contributions. 


\section{Modeling transition form factor}

Generic VDA representation (4.1) treats $(p z)$ and $z^{2}$ as independent variables, so we have no a priori reasons for a particular correlation of $x$ and $\sigma$ dependence in VDA. Thus, we try the simplest example: factorized models for VDAs, which result in factorized models for TMDAs, $\Phi(x, \sigma)=\varphi(x) \Phi(\sigma) \Rightarrow \Psi\left(x, k_{\perp}\right)=\varphi(x) \psi\left(k_{\perp}^{2}\right) / \pi$ in which the $x$-dependence and $k_{\perp}$-dependence appear in separate factors. Assuming a Gaussian dependence on $k_{\perp}$ for the TMDA results in a Gaussian dependence on $z_{\perp}$ for the IDA

$$
\Psi_{G}\left(x, k_{\perp}\right)=\frac{\varphi(x)}{\pi \Lambda^{2}} e^{-k_{\perp}^{2} / \Lambda^{2}} \Rightarrow \varphi_{G}\left(x, z_{\perp}\right)=\varphi(x) e^{-z_{\perp}^{2} \Lambda^{2} / 4} .
$$

One may argue that a Gaussian fall-off for large $z_{\perp}$ is too fast compared to exponential $\sim e^{-\left|z_{\perp}\right| m}$ behavior of a massive propagator. Some models with an exponential fall-off were given in Refs. $[5,6,7,8]$. Here we will consider an extreme $m=0$ limit of these models, when

$$
\Phi_{m=0}(x, \sigma ; \Lambda)=\varphi(x) \frac{e^{i \sigma / \Lambda^{2}-\varepsilon \sigma}}{i \Lambda^{2}} \text { giving } \varphi_{m=0}\left(x, z_{\perp}\right)=\frac{\varphi(x)}{1+z_{\perp}^{2} \Lambda^{2} / 4} .
$$

Note that the $z_{\perp}^{2}$ term of the $z_{\perp}$ expansion of $\varphi\left(x, z_{\perp}\right)$ in this model was adjusted to coincide with that of the exponential model, so that $\Lambda^{2}$ has the same meaning of the scale of $\bar{\psi} D^{2} \psi$ operator.

We can use these models now to calculate the higher twist contributions to the transition form factor. In the case of the Gaussian model (5.1), we have (for spin-1/2 quarks)

$$
F_{G}\left(Q^{2}\right)=\int_{0}^{1} \frac{d x}{x Q^{2}} \varphi(x)\left[1-\frac{\Lambda^{2}}{x Q^{2}}\left(1-e^{-x Q^{2} / \Lambda^{2}}\right)\right]
$$

Again, for large $Q^{2}$, Eq. (5.3) displays a power-like twist-4 correction and the term that corresponds to the region $k_{\perp}^{2} \geq x Q^{2}$ accumulating contributions of "invisible" operators with twist 6 and higher. Note that, despite of the $1 / x^{2}$ singular term in the integrand, the integral (5.3) is finite for $\varphi(x)$ as singular as $1 / x^{1-\alpha}$ with $\alpha>0$, which includes a flat DA (with $\alpha=1$ ). Furthermore, a formal $Q^{2} \rightarrow 0$ limit is finite: $F_{G}\left(Q^{2}=0\right)=f_{\pi} / 2 \Lambda^{2}$. In fact, $F\left(Q^{2}\right)$ is finite for $Q^{2}=0$ in any model with finite $\Psi\left(x, k_{\perp}=0\right)$. For the non-Gaussian $m=0$ model, we have

$$
F\left(Q^{2}\right)=\int_{0}^{1} \frac{d x}{x Q^{2}} \varphi(x)\left[1-\frac{\Lambda^{2}}{x Q^{2}}+2 K_{2}(2 \sqrt{x} Q / \Lambda)\right]
$$

where $\Lambda^{2}$ is again the scale characterizing the $\bar{q} D^{2} q$ matrix element.

To describe the BABAR data we use a flat DA $\varphi(x)=f_{\pi}$ with the scale $\Lambda_{G}^{2}=0.35 \mathrm{GeV}^{2}$ for the Gaussian model and $\Lambda_{m=0}^{2}=0.6 \mathrm{GeV}^{2}$ for the $m=0$ non-Gaussian model. Fitting the Belle data requires a more narrow DA $\varphi(x) \sim f_{\pi}(x \bar{x})^{0.4}$ with the scale $\Lambda_{G}^{2}=0.3 \mathrm{GeV}^{2}$ for the Gaussian model and a larger value $\Lambda_{m=0}^{2}=0.4 \mathrm{GeV}^{2}$ for the $m=0$ non-Gaussian model.

A close agreement of our models with the data (see Fig. 4) for a very wide range of $Q^{2}$ indicates that the "invisible contributions" correctly describe the physics behind the nontrivial shape of the experimental curves for $Q^{2} F\left(Q^{2}\right)$. Note also that one has $J=4.5$ for the DA $\varphi(x) \sim f_{\pi}(x \bar{x})^{0.4}$ used to describe the Belle data, which means that the curve $J\left(Q^{2}\right)$ is well below its asymptotic value even for $Q^{2} \sim 40 \mathrm{GeV}^{2}$. Within the standard pQCD picture, in which the form factor $F\left(Q^{2}\right)$ 

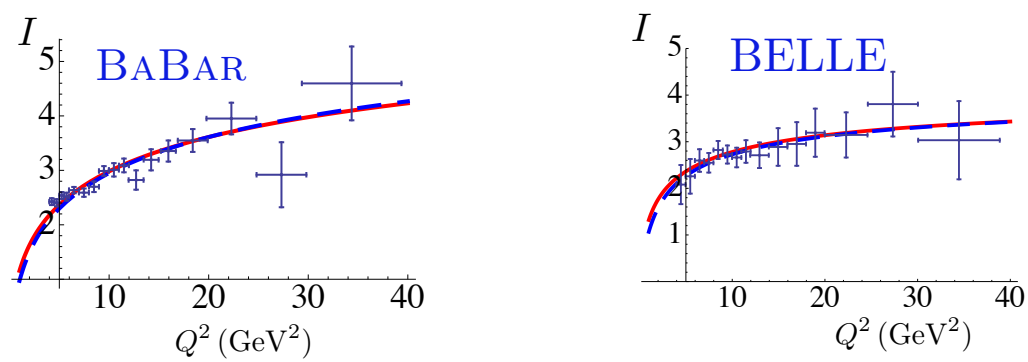

Figure 4: BABAR and Belle data compared to model curves described in the text.

is a sum of a few power-like terms, and given the size $\Lambda_{G}^{2}=0.3 \mathrm{GeV}^{2}$ or $\Lambda_{m=0}^{2}=0.4 \mathrm{GeV}^{2}$ of the scale involved, it is simply impossible to understand such a slow approach to the asymptotic value.

Another point is that in the region $Q^{2} \lesssim 10 \mathrm{GeV}^{2}$, where the BaBar and Belle data agree, the data may be described by models with both flat and $\sim(x \bar{x})^{0.4}$ behavior. In other words, the data alone cannot tell us what is the shape of the pion DA, even when the data are in a region of $Q^{2}$ by two orders of magnitude larger than $\Lambda_{\mathrm{QCD}}^{2}$.

\section{One-gluon-exchange diagram for the pion form factor}

Let us now apply the VDA formalism to the hard contribution for the pion electromagnetic (EM) form factor. The short-distance subprocess in this case contains one quark and one gluon propagator, with virtualities $x Q^{2}$ and $x y Q^{2}$, respectively (see Fig. 5a).

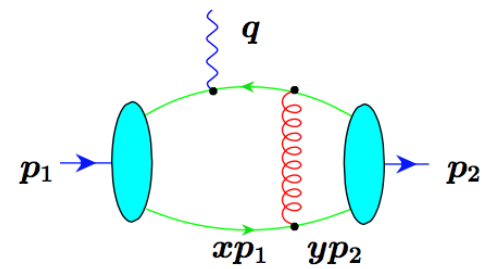

a)

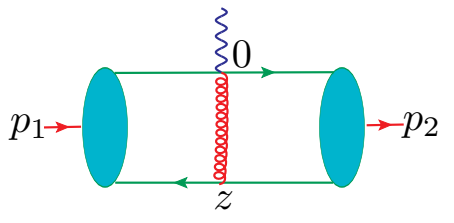

b)

Figure 5: Perturbative QCD one-gluon-exchange contribution to the pion EM form factor

In the twist-2 expression for spin-1/2 quarks, the denominator factor $x Q^{2}$ of the hard quark propagator is cancelled by the $x Q^{2}$ factor coming from the numerator trace, so that the final integral

$$
F_{\pi}^{\mathrm{as}(\mathrm{pQCD})}\left(Q^{2}\right)=\frac{8 \pi \alpha_{s}}{9} \int_{0}^{1} d x \int_{0}^{1} d y \frac{\varphi_{\pi}(x) \varphi_{\pi}(y)}{x y Q^{2}}=2 \frac{s_{0}}{Q^{2}} \frac{\alpha_{s}}{\pi}(J / 3)^{2}
$$

(where $s_{0}=4 \pi^{2} f_{\pi}^{2} \approx 0.7 \mathrm{GeV}^{2}$ ) is given by an expression which may be thought of as coming from a diagram where the hard quark propagator is shrunk into a point (see Fig. 5b). Again, the pQCD result diverges for flat DA $\varphi_{\pi}(x)=f_{\pi}$. Since the virtuality of exchanged gluon is $x y Q^{2}$, one may naively expect $x y Q^{2} \rightarrow x y Q^{2}+2 M^{2}$ when the transverse momentum is included. As we have learned, in the VDA approach such effects are desribed by formulas more complicated than that. 
To illustrate the VDA modifications, we consider the simplified diagram shown in Fig. 5b that has the same asymptotic expression as the full pQCD diagram Fig. 5a. It may be written as

$$
T\left(p_{1}, p_{2}\right)=a \int_{0}^{1} d x \int_{0}^{1} d y \int d^{4} z e^{-i x\left(p_{1} z\right)+i y\left(p_{2} z\right)} D_{c}(z) B\left(x, z^{2} / 4\right) B\left(y, z^{2} / 4\right) .
$$

in the coordinate representation (we denote $a=8 \pi \alpha_{s} / 9$ ). In terms of VDAs, we obtain

$$
F\left(Q^{2}\right)=a \int_{0}^{\infty} d \sigma_{1} \int_{0}^{\infty} d \sigma_{2} \int_{0}^{1} \Phi\left(x, \sigma_{1}\right) \int_{0}^{1} \Phi\left(y, \sigma_{2}\right) \frac{d x d y}{x y Q^{2}}\left[1-e^{-\left[i x y Q^{2}+\varepsilon\right] /\left(\sigma_{1}+\sigma_{2}\right)}\right],
$$

The first term in the square brackets does not depend on $\sigma_{1}, \sigma_{2}$ and produces the twist-2 expression. Using the definition (4.3) of IDA $\varphi\left(x, b_{\perp}\right)$ we arrive at

$$
F\left(Q^{2}\right)=\frac{a}{(2 \pi)^{2}} \int_{0}^{1} d x \int_{0}^{1} d y \int_{k_{\perp}^{2} \leq x y Q^{2}} \frac{d^{2} k_{\perp}}{x y Q^{2}} \int e^{i\left(k_{\perp} b_{\perp}\right)} \varphi\left(x, b_{\perp}\right) \varphi\left(y, b_{\perp}\right) d^{2} b_{\perp} .
$$

The $k_{\perp}^{2} \leq x y Q^{2}$ restriction converts into the Bessel function $J_{1}$ in the impact parameter space giving

$$
F\left(Q^{2}\right)=a \int_{0}^{1} d x \int_{0}^{1} d y \int_{0}^{\infty} \frac{d b_{\perp}}{\sqrt{x y Q^{2}}} J_{1}\left(b_{\perp} \sqrt{x y Q^{2}}\right) \varphi\left(x, b_{\perp}\right) \varphi\left(y, b_{\perp}\right) .
$$

Taking the factorized Ansatz with a Gaussian (G) or power-law (P) dependence on $b_{\perp}^{2}$

$$
\varphi_{G}\left(x, b_{\perp}\right)=\varphi(x) e^{-b_{\perp}^{2} \Lambda_{G}^{2} / 4}, \varphi_{P}\left(x, b_{\perp}\right)=\varphi(x) /\left(1+b_{\perp}^{2} \Lambda_{P}^{2} / 4\right)
$$

we obtain the following expressions

$$
\begin{aligned}
& F_{G}\left(Q^{2}\right)=a \int_{0}^{1} \frac{d x d y}{x y Q^{2}} \varphi(x) \varphi(y)\left[1-e^{-x y Q^{2} / 2 \Lambda_{G}^{2}}\right] \equiv a \frac{f_{\pi}^{2}}{Q^{2}} I_{G}\left(Q^{2}\right)=2 \frac{s_{0}}{Q^{2}} \frac{\alpha_{s}}{\pi} \frac{I_{G}\left(Q^{2}\right)}{9}, \\
& F_{P}\left(Q^{2}\right)=a \int_{0}^{1} \frac{d x d y}{x y Q^{2}} \varphi(x) \varphi(y)\left[1-2 \frac{x y Q^{2}}{\Lambda_{P}^{2}} K_{2}\left(2 \sqrt{x y} Q / \Lambda_{P}\right)\right] \equiv a \frac{f_{\pi}^{2}}{Q^{2}} I_{P}\left(Q^{2}\right) .
\end{aligned}
$$

Just like in the transition form factor case, these integrals are finite for $Q^{2}=0$, with the values $F_{G}\left(Q^{2}=0\right)=a f_{\pi}^{2} / 2 \Lambda_{G}^{2}, F_{P}\left(Q^{2}=0\right)=a f_{\pi}^{2} / \Lambda_{P}^{2}$.

Taking for definitness the scales $\Lambda_{G}^{2}=0.35 \mathrm{GeV}^{2}$ and $\Lambda_{P}^{2}=0.6 \mathrm{GeV}^{2}$ of the same size as those that were fitting the BaBar transition form factor data, we plot the ratio $I\left(Q^{2}\right) / 9$ for both cases, see Figs. 6, 7. To show the sensitivity to the shape of the pion DA, we use 3 different choices for $\varphi(x)$, namely, flat, "root", and asymptotic. Notice that "1" on these plots would correspond to $\mathrm{pQCD}$ prediction with asymptotic DA. One can see from Fig. 6 that it requires pretty large $Q^{2} \gtrsim 20 \mathrm{GeV}^{2}$ to clearly discriminate between the 3 curves. To emphasize this point, we repeat the plots on Fig. 7, restricting the range of $Q^{2}$ to experimentally accessible values of $Q^{2} \lesssim 10 \mathrm{GeV}^{2}$. One can see that the 3 curves are practically indistinguishable in this region. To understand this outcome, we note that the curves are still rising with $Q^{2}$ and are almost linear. Thus, we conclude that the average gluon virtuality $\left\langle x y Q^{2}\right\rangle$ in this region is practically constant, e.g. in Gaussian case $\left\langle 1 /\left(x y Q^{2}\right)\right\rangle=\left[2 \Lambda_{G}^{2}+\langle x\rangle\langle y\rangle Q^{2} / 2+\ldots\right]^{-1}=\left[2 \Lambda_{G}^{2}+Q^{2} / 8+\ldots\right]^{-1}$. It starts to become visibly proportional to $Q^{2}$ only when the curves on Fig. 6 flatten, i.e. well above $Q^{2} \sim 10 \mathrm{GeV}^{2}$.

In other words, we see no chances to detect experimentally the $\sim 1 / Q^{2}$ perturbative QCD prediction for the "hard" gluon-exchange contribution. Furthermore, we observe that the shape 

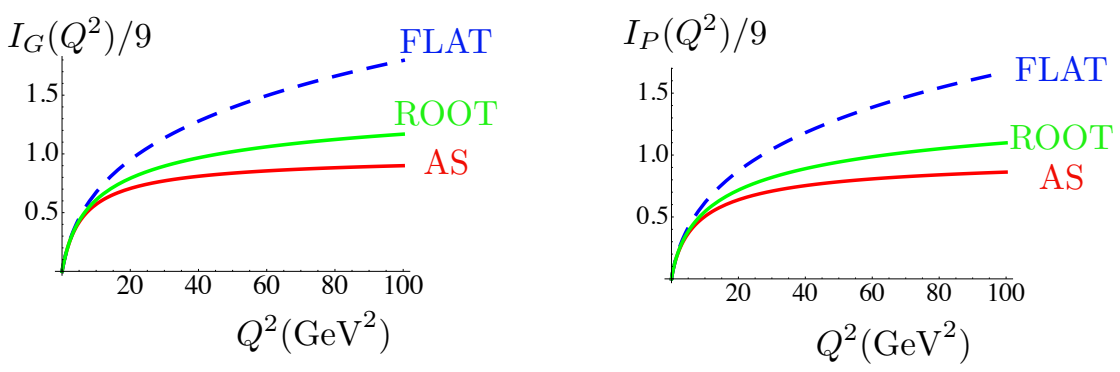

Figure 6: Functions $I\left(Q^{2}\right)$ for Gaussian (left) and power-like (right) IDA models for $Q^{2}$ up to $100 \mathrm{GeV}^{2}$.

of the pion DA is not important as far as $Q^{2} \lesssim 10 \mathrm{GeV}^{2}$ : the result for the flat DA in this region is practically the same as for the asymptotic DA. Moreover, because the asymptotic behavior sets very slowly, the absolute value of $I\left(Q^{2}\right) / 9$ is well below "1" corresponding to the formal pQCD prediction, approaching just a half of it for the highest $Q^{2} \sim 10 \mathrm{GeV}^{2}$ reachable at Jefferson Lab.

Numerically, using $2 s_{0} \approx 1.3 \mathrm{GeV}^{2}$ and assuming a "frozen" value $\alpha_{s} / \pi=0.1$, we have $Q^{2} F_{\pi}^{\text {gluon exchange }}\left(Q^{2}\right)=0.13\left[I\left(Q^{2}\right) / 9\right] \mathrm{GeV}^{2}$. Thus, our estimate gives $Q^{2} F_{\pi}^{\text {gl. ex. }}\left(Q^{2}\right) \lesssim 0.04 \mathrm{GeV}^{2}$ for $Q^{2}=2.45 \mathrm{GeV}^{2}$ or more than 10 times below the existing JLab measurement [19]. Though this outcome doubles to $0.08 \mathrm{GeV}^{2}$ for $Q^{2}=10 \mathrm{GeV}^{2}$, it still does not look significant.
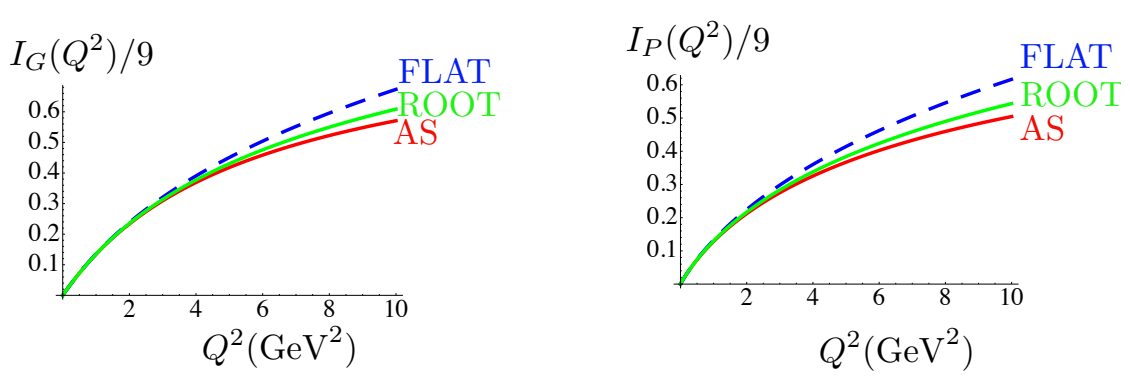

Figure 7: Functions $I\left(Q^{2}\right)$ for Gaussian (left) and power-like (right) IDA models for $Q^{2}$ up to $10 \mathrm{GeV}^{2}$.

One may ask: if the gluon exchange contribution is so small, what is the mechanism explaining observed pion EM form factor behavior? The answer was given long time ago [20]: it is the Feynman mechanism, corresponding (in the light-front terminology) to the Drell-Yan [3] overlap of soft wave functions. However, this is a subject of a different talk and, hopefully, another direction for applications of the VDA formalism!

\section{Acknowledgements}

This work is supported by Jefferson Science Associates, LLC under U.S. DOE Contract No. DE-AC05-06OR23177 and by U.S. DOE Grant \#DE-FG02-97ER41028.

\section{References}

[1] A. Radyushkin, Deep Elastic Processes of Composite Particles in Field Theory and Asymptotic Freedom, JINR report P2-10717 (1977) [hep-ph/ 0410276 ]. 
[2] G. P. Lepage and S. J. Brodsky, Exclusive Processes in Quantum Chromodynamics: Evolution Equations for Hadronic Wave Functions and the Form-Factors of Mesons, Phys. Lett. B87 (1979) 359-365.

[3] S. Drell and T.-M. Yan, Connection of Elastic Electromagnetic Nucleon Form-Factors at Large $Q^{2}$ and Deep Inelastic Structure Functions Near Threshold, Phys.Rev.Lett. 24 (1970) 181-185.

[4] G. P. Lepage and S. J. Brodsky, Exclusive Processes in Perturbative Quantum Chromodynamics, Phys. Rev. D22 (1980) 2157.

[5] A. V. Radyushkin, Virtuality Distributions in Application to $\gamma \gamma^{*} \rightarrow \pi^{0}$ Transition Form Factor at Handbag Level, Phys. Lett. B735 (2014) 417-425, [arXiv: 1404 . 7032 ].

[6] A. V. Radyushkin, Virtuality Distributions and $\gamma \gamma^{*} \rightarrow \pi^{0}$ Transition Form Factor at Handbag Level, Few Body Syst. 56 (2015), no. 6-9 287-293.

[7] A. V. Radyushkin, Virtuality Distributions and Pion Transition Form Factor, Int. J. Mod. Phys. Conf. Ser. 37 (2015) 1560050.

[8] A. V. Radyushkin, Virtuality and Transverse Momentum Dependence of Pion Distribution Amplitude, Report JLAB-THY-15-2145 (2015) [arXiv:1510.02517].

[9] A. V. Efremov and A. V. Radyushkin, Factorization and Asymptotical Behavior of Pion Form-Factor in QCD, Phys. Lett. B94 (1980) 245-250.

[10] A. Efremov and A. Radyushkin, High momentum transfer processes in QCD, JINR report E2-11535 (1978).

[11] S. V. Mikhailov and A. V. Radyushkin, Nonlocal Condensates and QCD Sum Rules for Pion Wave Function, JETP Lett. 43 (1986) 712. [Pisma Zh. Eksp. Teor. Fiz.43,551(1986)].

[12] S. V. Mikhailov and A. V. Radyushkin, The Pion wave function and QCD sum rules with nonlocal condensates, Phys. Rev. D45 (1992) 1754-1759.

[13] V. M. Braun, S. Collins, M. Göckeler, P. Pérez-Rubio, A. Schäfer, R. W. Schiel, and A. Sternbeck, Second Moment of the Pion Light-cone Distribution Amplitude from Lattice QCD, Phys. Rev. D92 (2015), no. 1 014504, [arXiv:1503.03656].

[14] V. M. Braun and I. E. Filyanov, QCD Sum Rules in Exclusive Kinematics and Pion Wave Function, Z. Phys. C44 (1989) 157. [Yad. Fiz.50,818(1989)].

[15] BaBar Collaboration, B. Aubert et al., Measurement of the $\gamma \gamma^{*} \rightarrow \pi^{0}$ transition form factor, Phys. Rev. D80 (2009) 052002, [arXiv: 0905 .4778].

[16] Belle Collaboration, S. Uehara et al., Measurement of $\gamma \gamma^{*} \rightarrow \pi^{0}$ transition form factor at Belle, Phys. Rev. D86 (2012) 092007, [arXiv: 1205.3249 ].

[17] A. V. Radyushkin, Shape of Pion Distribution Amplitude, Phys. Rev. D80 (2009) 094009, [arXiv:0906.0323].

[18] I. V. Musatov and A. V. Radyushkin, Transverse momentum and Sudakov effects in exclusive QCD processes: $\gamma^{*} \gamma \pi^{0}$ form-factor, Phys. Rev. D56 (1997) 2713-2735, [hep-ph/9702443].

[19] Jefferson Lab F(pi)-2 Collaboration, T. Horn et al., Determination of the Charged Pion Form Factor at $Q^{2}=1.60$ and $2.45(\mathrm{GeV} / \mathrm{c})^{2}$, Phys. Rev. Lett. 97 (2006) 192001, [nucl-ex/ 0607005 ].

[20] V. A. Nesterenko and A. V. Radyushkin, Sum Rules and Pion Form-Factor in QCD, Phys. Lett. B115 (1982) 410 . 